\title{
Síntese e caracterização de argila esmectita Zn-estevensita
}

\section{(Synthesis and characterization of smectite clay Zn-stevensite)}

\author{
E. A. Hildebrando ${ }^{1}$, M. G. Silva-Valenzuela ${ }^{2,3}$, R. F. Neves ${ }^{4}$, F. R. Valenzuela-Diaz ${ }^{2}$ \\ ${ }^{1}$ Laboratório de Materiais Cerâmicos, Faculdade de Engenharia de Materiais, Universidade Federal do Pará, \\ Campus Universitário de Marabá, Marabá, PA 68505-080 \\ ${ }^{2}$ Laboratório de Matérias-Primas Particuladas e Sólidos Não Metálicos, Departamento de Engenharia \\ Metalúrgica e de Materiais, Escola Politécnica da Universidade de S. Paulo, S. Paulo, SP \\ ${ }^{3}$ Centro Universitário Estácio Radial de S. Paulo \\ ${ }^{4}$ Faculdade de Engenharia Química, Universidade Federal do Pará, Cidade Universitária, Belém, PA \\ edemarino@ufpa.br,gracavalenzuela@gmail.com,rfn@ufpa.br,frrvdiaz@usp.br
}

\begin{abstract}
Resumo
Argila do grupo das esmectitas foi obtida com êxito em baixa temperatura de processamento por via hidrotérmica utilizando como precursores metassilicato de sódio, nitrato de zinco e uréia. Durante a etapa de síntese a composição molar da mistura reacional e a temperatura permaneceram constantes, variando-se o tempo de reação. As amostras sintetizadas foram caracterizadas por difração de raios X, microscopia eletrônica de varredura, espectroscopia de refletância difusa no infravermelho com transformada de Fourier, análise térmica diferencial e gravimétrica, análise de adsorção gasosa de nitrogênio pelo método BET e capacidade de troca de cátions. Os resultados evidenciam que com a metodologia empregada obtêm-se Zn-estevensita apresentando uma área superficial total na faixa de 171,6 a $203,4 \mathrm{~m}^{2} / \mathrm{g}$ e boa cristalinidade; sendo esta a única fase presente no produto sintetizado após reação estática a $90{ }^{\circ} \mathrm{C}$ por um período de 44 a 138 h, indicando ser o aumento no tempo de síntese um parâmetro importante para o processo de cristalização da argila.
\end{abstract}

Palavras-chave: esmectita, argila sintética, estevensita.

\begin{abstract}
Clay of the smectite group was successfully obtained at a low temperature hydrothermal processing using sodium metasilicate, zinc nitrate and urea as precursor materials. During the synthesis step, the molar composition of the mixture and the temperature remained constant varying the reaction time. The synthesized samples were characterized by X-ray diffraction, scanning electron microscopy, diffuse reflectance Fourier Transform infrared spectroscopy, differential thermal analysis and thermogravimetry, cation exchange capacity (CEC) and BET surface area measurements. The results show that in the methodology utilized is obtained Znstevensite with specific surface area of 171.6-203.4 $\mathrm{m}^{2} / \mathrm{g}$ and good crystallinity as present single phase in the reaction product after static synthesis at $90{ }^{\circ} \mathrm{C}$ for 44 to $138 \mathrm{~h}$, indicating that, the increased synthesis time is an important parameter for crystallization process of the clay.
\end{abstract}

Keywords: smectite, sintetic clay, stevensite.

\section{INTRODUÇÃO}

Esmectitas são filossilicatos ou silicatos em camadas constituídas por duas folhas de tetraedros "ensanduichando" uma folha central de octaédros, unidas entre si por oxigênios comuns às mesmas, apresentando deste modo uma estrutura cristalina classificada como do tipo 2:1 [1]. É um dos mais importantes grupos de argilas encontrados em solos e sedimentos, e certamente um dos mais difíceis para se estudar [2]. Suas características específicas, como elevada área específica, capacidade de troca de cátions e propriedades de hidratação, conferem a esta família de argilominerais diversos interesses industriais e uma grade variedade de aplicações dentre as quais se destacam a sua utilização em nanocompósitos, na indústria cerâmica, em formulação de cosméticos, no tratamento de rejeitos, na descontaminação de solos, entre outras [3,4]. Apesar deste tipo de argila ser muito comum na natureza, um dos principais motivos para se buscar esmectitas sintéticas, é que as suas ocorrências em depósitos naturais apresentam vários inconvenientes, dentre os quais, a existência de impurezas; o que desencadeia ao argilomineral de interesse uma qualidade duvidosa e uma quantidade insignificante ou muito pequena em sua forma natural, limitando desta maneira, o seu uso em potencial para determinadas aplicações industriais $[5,6]$; com o uso de argilas sintéticas é possível evitar esses problemas. Assim, o conhecimento obtido a partir das tecnologias de síntese pode contribuir para uma melhor compreensão dos mecanismos de formação dos argilominerais, permitindo de tal modo controlar a quantidade de substituições isomórficas nos 
sólidos sintetizados levando desta forma a modificações na composição e propriedade dos mesmos, e consequentemente, ao surgimento de novas aplicações [6].

Durante os últimos 60 anos, inúmeros experimentos de síntese de esmectitas foram realizados. Vários artigos de revisão discutem as abordagens para a síntese de esmectitas sob diferentes conjuntos de condições (temperatura e pressão baixa, tratamento hidrotérmico moderado e extremo, e reações na presença de fluoreto) $[2,5,6]$. De acordo com o corrente estado da arte a síntese deste argilomineral é tecnicamente difícil; pois, na grande maioria das vezes, exige um processo hidrotermal prolongado realizado a pressões e temperaturas relativamente altas [7]. No entanto, é fato que alguns argilominerais esmectíticos, como por exemplo, a saponita e a estevensita, podem ser sintetizados em baixas temperaturas e pressões via hidrólise da uréia onde o ponto chave para preparação de ambas esmectitas é o controle do $\mathrm{pH}$ [7-9].

A estevensita encontra-se classificada no subgrupo das esmectitas trioctaédricas. É representada pela seguinte fórmula estrutural , $\mathrm{N}_{\mathrm{x} / \mathrm{Z}}^{\mathrm{Z}+}\left[\mathrm{M}_{6-\mathrm{x}}^{2+} \Delta_{\mathrm{x}}\right]\left[\mathrm{Si}_{8}\right] \mathrm{O}_{20}(\mathrm{OH})_{4} \cdot \mathrm{nH}_{2} \mathrm{O}$, onde $M$ corresponde ao cátion divalente ( $\mathrm{Zn}^{2+}, \mathrm{Co}^{2+}$, entre outros) localizado na folha octaédrica, $N$ o cátion que ocupa o espaço interplanar das camadas $2: 1$ possuindo um espaçamento basal em torno de 13 a $15 \AA$, $n$ é o número de moléculas de água que separam as camadas e $\Delta_{\mathrm{x}}$ são vacâncias localizadas nas camadas [2,7]. Sua aplicação encontra-se até então limitada ao uso em catálise heterogênea em reações de hidrodessulfurização; no entanto, sua utilização como nanocargas para obtenção de nanocompósitos também tem sido estudada demonstrando perspectivas promissoras [7, 10]. Dessa forma, o objetivo deste trabalho foi produzir argila sintética do grupo das esmectitas (Zn-estevensita), alterando-se em parte os procedimentos relatados na literatura, e implementando aos mesmos modificações essencialmente no que diz respeito ao processo de síntese e aos precursores empregados.

\section{MATERIAIS E MÉTODOS}

Os reagentes utilizados no processo de síntese das amostras foram todos de pureza analítica. Como fonte de silício e zinco foram empregados respectivamente metassilicato de sódio pentahidratado $\left(\mathrm{Na}_{2} \mathrm{SiO}_{3} .5 \mathrm{H}_{2} \mathrm{O}\right)$ e nitrato de zinco hexahidratado $\left(\mathrm{Zn}\left(\mathrm{NO}_{3}\right)_{2} \cdot 6 \mathrm{H}_{2} \mathrm{O}\right)$; para completar a mistura reacional utilizou-se uréia $\left(\mathrm{CO}\left(\mathrm{NH}_{2}\right)_{2}\right)$ e ácido nítrico concentrado $\left(\mathrm{HNO}_{3}\right)$.

Preparação das amostras: o material foi sintetizado seguindo basicamente o método de decomposição da uréia, o qual é descrito em detalhes [7-9]. Contudo, algumas modificações foram efetuadas, principalmente no que concerne a fonte de silício, e as condições de reação, acrescentando ao método de preparação da amostra, o processo hidrotermal brando em sistema estático. Então, $110 \mathrm{~mL}$ de solução contendo $10,0 \mathrm{~g}$ de $\mathrm{Zn}\left(\mathrm{NO}_{3}\right)_{2} \cdot 6 \mathrm{H}_{2} \mathrm{O}$; $20,0 \mathrm{~g}$ de $\mathrm{Na}_{2} \mathrm{SiO}_{3} .5 \mathrm{H}_{2} \mathrm{O}$ e $10,0 \mathrm{~g}$ de $\mathrm{CO}\left(\mathrm{NH}_{2}\right)_{2}$; com um pH inicial entre 1-2 (ajustado com $\mathrm{HNO}_{3}$ ) foi preparada em um béquer. Em seguida a mesma foi acondicionada em um vaso de reação de $305 \mathrm{~cm}^{3}$, o qual foi colocado em estufa a $90{ }^{\circ} \mathrm{C}$ em processamento sem agitação. Os produtos formados foram lavados, através de filtração com água destilada, e em seguida submetidos à secagem a $110{ }^{\circ} \mathrm{C}$ por $24 \mathrm{~h}$, sendo denominados pela seguinte nomenclatura: ES1, ES2 e ES3 correspondentes aos tempos de síntese de 44, 90 e $138 \mathrm{~h}$, respectivamente.

Caracterização dos materiais: as amostras obtidas como produto de síntese foram caracterizadas por meio de: difração de raios X (DRX) em difratômetro X'Pert PRO PANalytical, com goniômetro PW 3050/60 e anodo de cobre, varredura de $5^{\circ}$ a $75^{\circ}$ em $2 \Theta, 45 \mathrm{kV}$ e $35 \mathrm{~mA}$; microscopia eletrônica de varredura (MEV) com imagens obtidas por meio de elétrons secundários em microscópio Philips XL30 e, quando necessário, microanálise por espectroscopia dispersiva de energia (EDS); espectroscopia de infravermelho (FTIR) em um equipamento Thermo-Nicolet Magna 560 com varredura de 400 a $4000 \mathrm{~cm}^{-1}$ e resolução de $4 \mathrm{~cm}^{-1}$, aplicando-se a técnica de refletância difusa (DRIFT); análise térmica diferencial e gravimétrica em equipamento PL Thermal Sciences, com analisador térmico simultâneo STA 1000/1500 Stanton Redcroft Ltd., com cadinho de alumina para $15 \mathrm{mg}$ de amostra, com varredura na faixa de temperatura ambiente até $1050{ }^{\circ} \mathrm{C}$, e taxa de aquecimento $20^{\circ} \mathrm{C} \cdot \mathrm{min}^{-1}$; a avaliação de área específica foi pelo método BET multiponto, a partir dos dados de isotermas de adsorção de nitrogênio a $77 \mathrm{~K}$ na faixa de pressões de vapor entre 0,01 a 0,99 em um equipamento Micromeritics ASAP 2010; medida da capacidade de troca catiônica (CTC) pelo método de acetato de amônio [11].

\section{RESULTADOS E DISCUSSÃO}

Observa-se nos difratogramas da Fig. 1 que todas as amostras sintetizadas apresentam a reflexão $(060)$ na posição $1,53 \AA\left(2 \theta=60,6^{\circ}\right)$ característico de argilas trioctaédricas com estrutura do tipo esmectita $[8,12]$; os picos em $2 \theta=$ $5,8^{\circ}(15,16 \AA), 19,5^{\circ}(4,53 \AA)$ e $33,9^{\circ}(2,65 \AA)$ sugerem a formação de produtos sintéticos com estrutura da estevensita como única fase e se encontram de acordo com o relatado para Zn-estevensita [13]. Para tempos de síntese maiores que $44 \mathrm{~h}$ os materiais produzidos (ES2 e ES3) apresentam um aumento na intensidade dos picos observado pela melhor definição das reflexões em $2 \theta=19,5^{\circ}$ e $33,9^{\circ}$ o que denota uma melhoria na cristalinidade.

As imagens de MEV das amostras ES1, ES2 e ES3 são apresentadas nas Figs. 2a-c. As imagens são similares, apresentando uma distribuição de tamanho de grãos constituídos por aglomerados de pequenas partículas de perfil regular e uniforme, que se unem através de sua superfície formando um agregado maior, demonstrando, desta forma, um comportamento similar ao encontrado por outros autores [13] para estrutura típica de argilas do grupo das esmectitas. Pelo espectro de EDS (Fig. 2d), verificase que o material sintetizado apresenta, em uma análise 

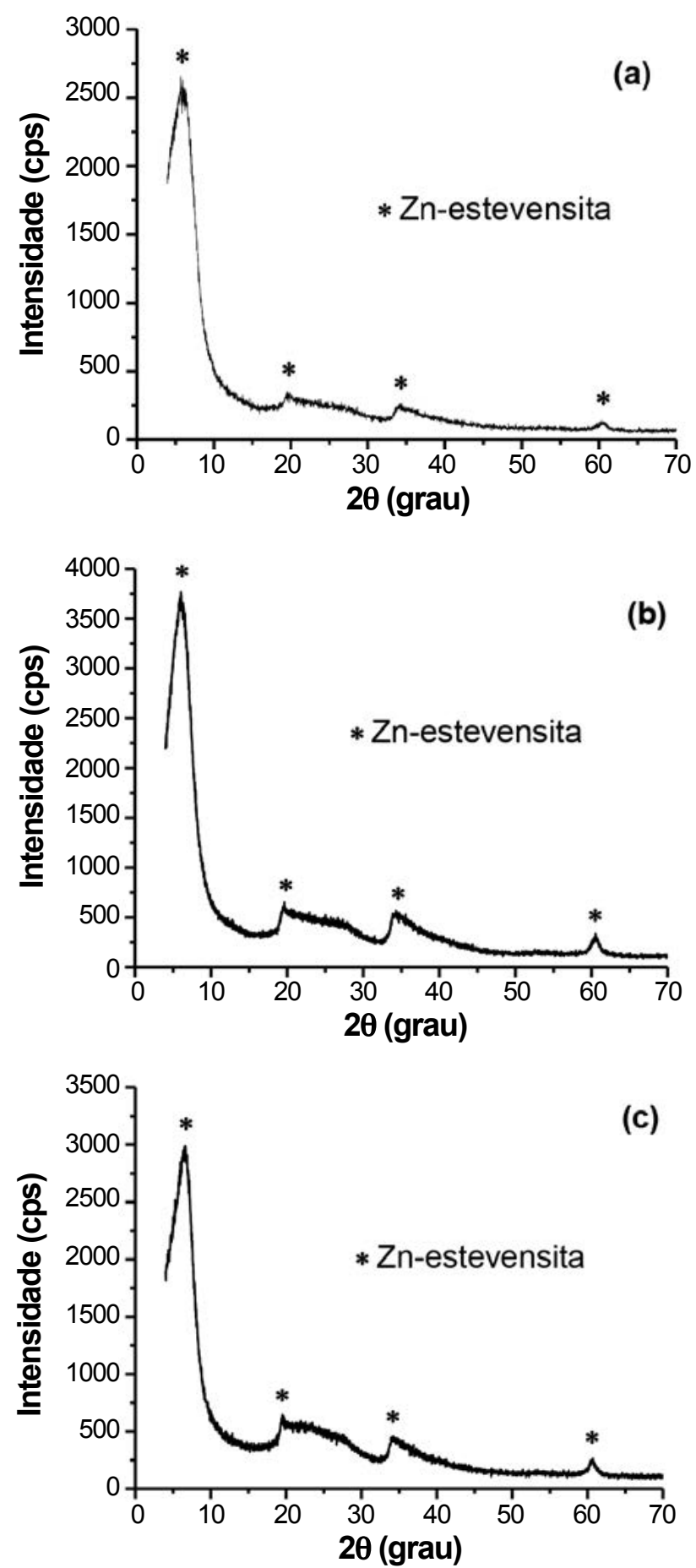

Figura 1: Difratogramas de raios X das amostras sintetizadas: (a) ES1; (b) ES2; (c) ES3

[Figure 1: X-ray diffraction patterns of the samples synthesized: (a) ES1; (b) ES2; (c) ES3.]

qualitativa aproximada de sua composição química, silício, zinco, sódio e oxigênio, como os principais elementos que fazem parte da constituição estrutural da argila obtida durante o processo de síntese, os quais são característicos para Zn-estevensita.

As análises de infravermelho foram realizadas (Figs. 3a-c) a fim de se observar a influência do tempo de síntese no processo de cristalização da argila. Os espectros de FTIR são semelhantes. Claramente se visualiza uma banda larga na região em torno de $3400 \mathrm{~cm}^{-1}$, que se encontra relacionada a vibrações que correspondem ao estiramento $\mathrm{O}-\mathrm{H}$ oriundo de moléculas de água dissociadas e não dissociadas. O pico agudo identificado a $1650 \mathrm{~cm}^{-1}$ constitui a deformação do estiramento $\mathrm{O}-\mathrm{H}$ corroborado pela análise anterior. O pico pouco intenso em torno de $3640 \mathrm{~cm}^{-1}$ está associado ao estiramento de ligações do tipo $\mathrm{Zn}-\mathrm{OH}[9,14]$ e é característico de argilas do grupo das esmectitas. Na região de baixa energia o espectro mostra um pico a 1060 $\mathrm{cm}^{-1}$ referente a vibrações de $\mathrm{Si}-\mathrm{O}$ e $\mathrm{Si}-\mathrm{O}-\mathrm{Si}$; o pico próximo a $671 \mathrm{~cm}^{-1}$ é um indicativo de argilas trioctraedricas, neste caso a $\mathrm{Zn}$-stevensita, e é principalmente devido a ligações do tipo $\mathrm{Zn}_{3} \mathrm{OH}$ [14]; já o pico a $521 \mathrm{~cm}^{-1}$ pode ser atribuído a ligações do tipo Si-O-Zn. Existem, no entanto, apesar da similaridade, algumas diferenças nos espectros vibracionais das amostras que podem estar relacionadas com a pureza e a cristalinidade. A primeira delas pode ser obsevada pela intensidade da banda na região $3650-3300 \mathrm{~cm}^{-1}$, a qual se torna mais difusa para a amostra ES1 indicando assim uma fraca coordenação do grupo hidroxila da folha octaédrica com o íon metálico zinco; outra diferença se refere à banda na faixa $1300-1430 \mathrm{~cm}^{-1}$ que se encontra relacionada a produtos de reação de uréia e nitrato não dissolvido $[12,14]$; verifica-se que o pico a $1430 \mathrm{~cm}^{-1}$, relacionado ao íon $\mathrm{NH}_{4}^{+}$, resultante da decomposição da uréia, tende a desaparecer nas amostras ES2 e ES3, o que caracteriza uma melhor formação dos produtos de síntese em função de uma maior interação entre os reagentes a partir da elevação do período reacional concordando desta forma com a difração de raios X.

A partir das curvas de análise térmica dos produtos sintetizados, Figs. 4a-c, observam-se picos endotérmicos próximos a $90^{\circ} \mathrm{C}$ e a $612-638^{\circ} \mathrm{C}$, e outro exotérmico em $\sim 799$ ${ }^{\circ} \mathrm{C}$, apresentando-se estes bem definidos, os quais possuem semelhanças para a Zn-estevensita, conforme descrito [14-16]. O primeiro pico endotérmico, com uma perda de massa em torno de $10 \%$, está associado à eliminação de água adsorvida (desidratação) e o segundo pico, com perda de aproximadamente $3 \%$, é característico da desidroxilação da esmectita. A temperatura de ocorrência deste segundo pico endotérmico aumenta gradativamente $(612,625$ e 638 ${ }^{\circ} \mathrm{C}$ ), refletindo assim [14] uma maior estabilidade térmica da amostra, podendo este evento estar diretamente relacionado com o aumento do tempo de síntese. Picos exotérmicos a $799{ }^{\circ} \mathrm{C}$ e acima de $1000{ }^{\circ} \mathrm{C}$, que não acompanham uma perda de peso significativa, correspondem a processos de transformações de fase, neste caso, da estevensita para seus polimorfos $\left(\mathrm{Zn}_{2} \mathrm{SiO}_{4}\right)$; estas temperaturas de recristalização dependem muito da composição catiônica inicial da argila, situando-se, no caso do primeiro pico, na faixa de $870{ }^{\circ} \mathrm{C}$ para Mg-estevensita, a $790^{\circ} \mathrm{C}$ para Zn-estevensita e a $710^{\circ} \mathrm{C}$ para Co-estevensita [16]. O pico exotérmico em $322^{\circ} \mathrm{C}$ pode ser atribuído a reações com alguns dos precursores (nitrato e uréia), o que denota que o gel inicial não foi direcionado em sua totalidade de modo a ser transformado completamente em argila, observa-se, entretanto, que para tempos de síntese 

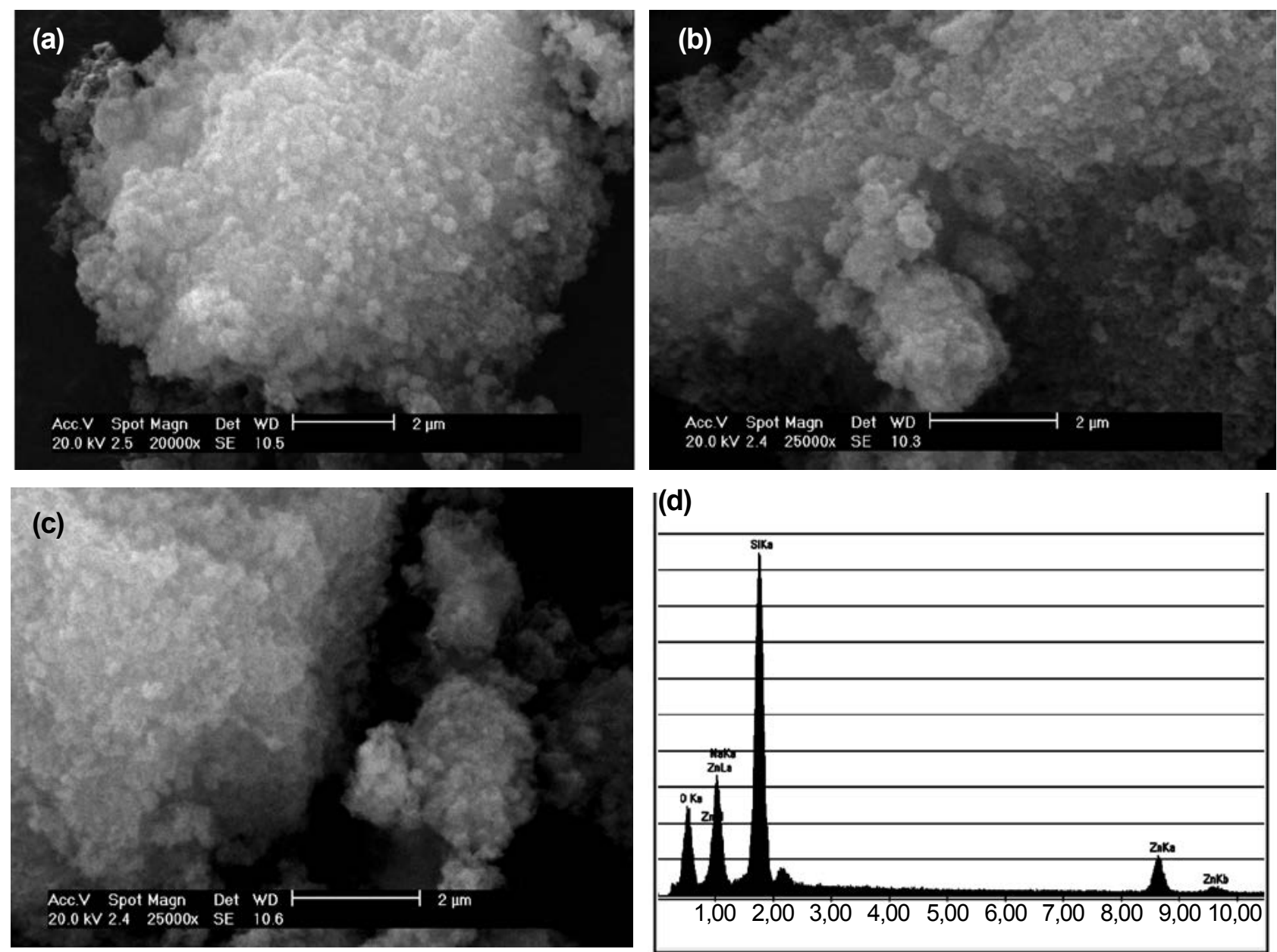

Figura 2: Micrografias obtidas em microscópio eletrônico de varredura das amostras: (a) ES1, (b) ES2, (c) ES3; e (d) espectro de EDS da amostra ES1.

[Figure 2: SEM micrographs of the samples: (a) ES1, (b) ES2, (c) ES3; and (d) XEDS spectrum of ES1 sample.]

mais elevados este pico tende a desaparecer, o que pode ser um indicativo de um melhor rendimento dos produtos reacionais.

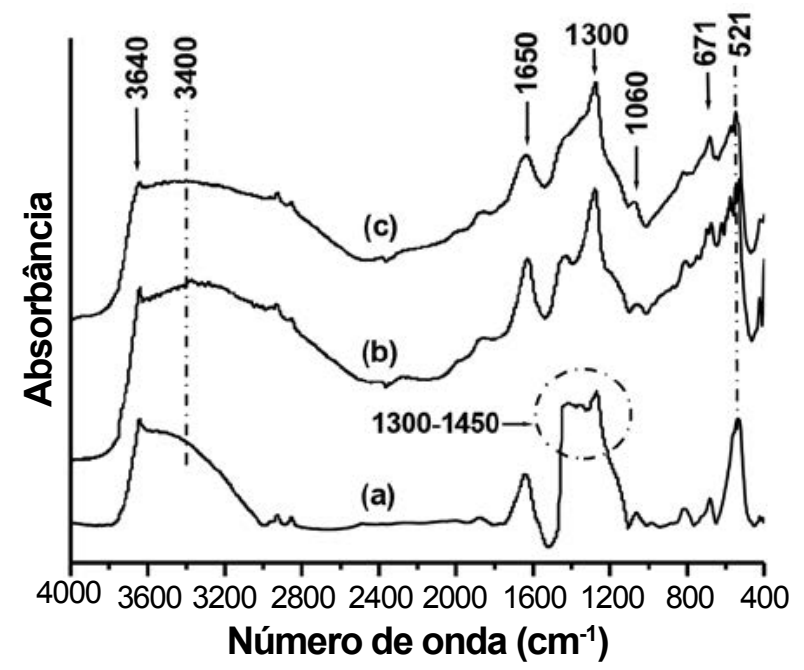

Figura 3: Espectros de DRIFT: (a) ES1; (b) ES2; (c) ES3. [Figure 3: DRIFT spectra: (a) ES1; (b) ES2; (c) ES3.]
A capacidade de troca catiônica (CTC) da estevensita está relacionada muito mais à deficiência de cátions na folha octaédrica (vacância) do que com as substituições destes mesmos cátions (substituições isomórficas) [15]. Verificase na Tabela I um pequeno aumento nos valores de CTC dos produtos sintetizados para tempos de reação mais elevados corroborando desta forma com as curvas de difração que apresentaram aumento na cristalinidade das amostras ES2 e ES3.

Todas as amostras apresentam valores de CTC compatíveis com filossilicato do tipo estevensita; os resultados foram considerados favoráveis, quando comparados com dados relatados pela literatura, e se encontram dentro da faixa esperada para este tipo de argila sendo ela natural $[17,18]$ ou sintética $[7,15]$.

A Fig. 5 mostra as isotermas de adsorção/dessorção das amostras sintetizadas, apresentando as mesmas segundo a classificação recomendada pela IUPAC, comportamentos de isoterma do tipo IV, típica de materiais mesoporosos, cuja principal característica é a existência de uma histerese, e a ausência de limitação de adsorção de nitrogênio em valores elevados de $P / P_{0}$ [19]. Com relação ao ciclo de histerese, 

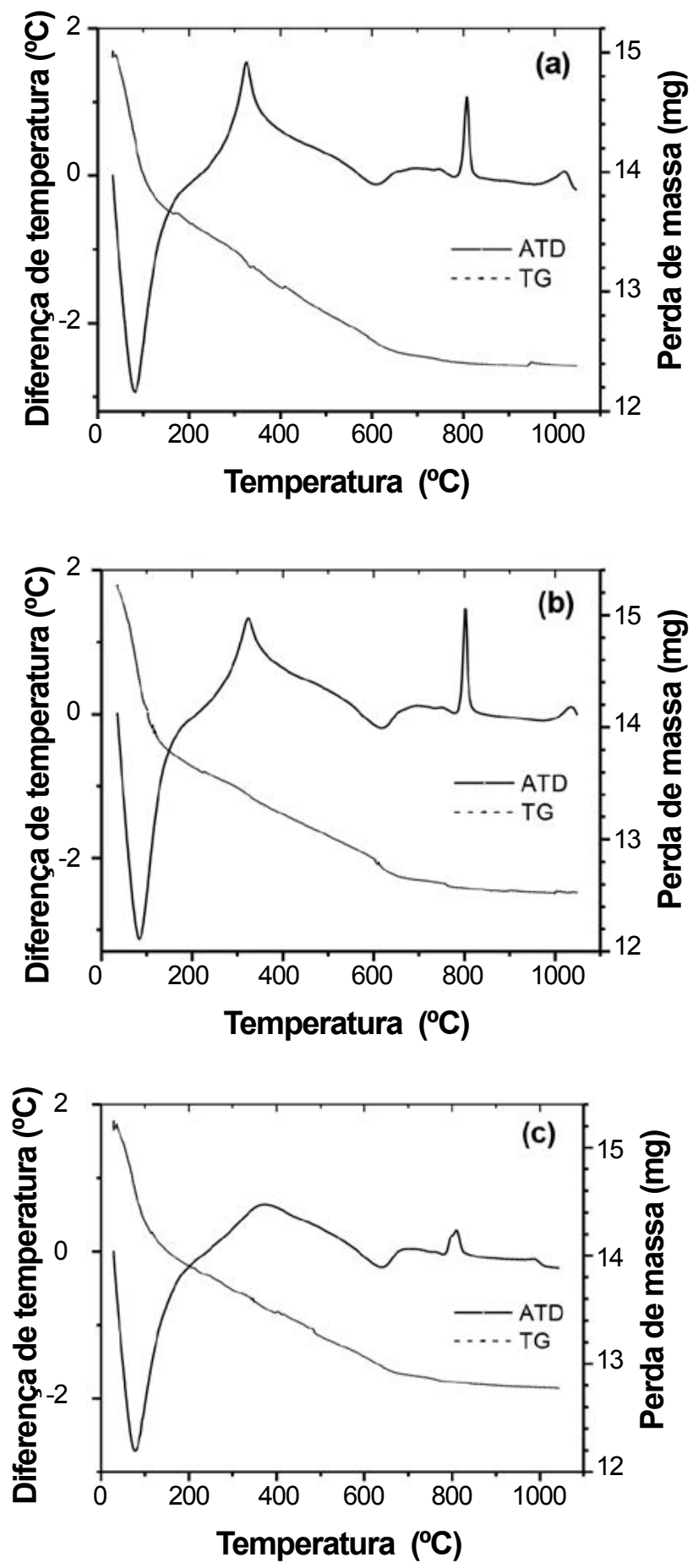

Figura 4: Curvas de ATD/TG das amostras: (a) ES1; (b) ES2; (c) ES3.

[Figure 4: ATD/TG curves of samples: (a) ES1; (b) ES2; (c) ES3.]

Tabela I - CTC das amostras sintetizadas a $90{ }^{\circ} \mathrm{C}$ e valores de referência.

[Table I - CEC of the samples synthesized at $90{ }^{\circ} \mathrm{C}$ and references.]

\begin{tabular}{llllllll}
\hline Amostras & ES1 & ES2 & ES3 & [7] & [15] & [17] & {$[18]$} \\
\hline CTC (mEq/100g) & 63,8 & 67,4 & 70,1 & $30-50$ & 41 & 41 & 76,5 \\
\hline
\end{tabular}
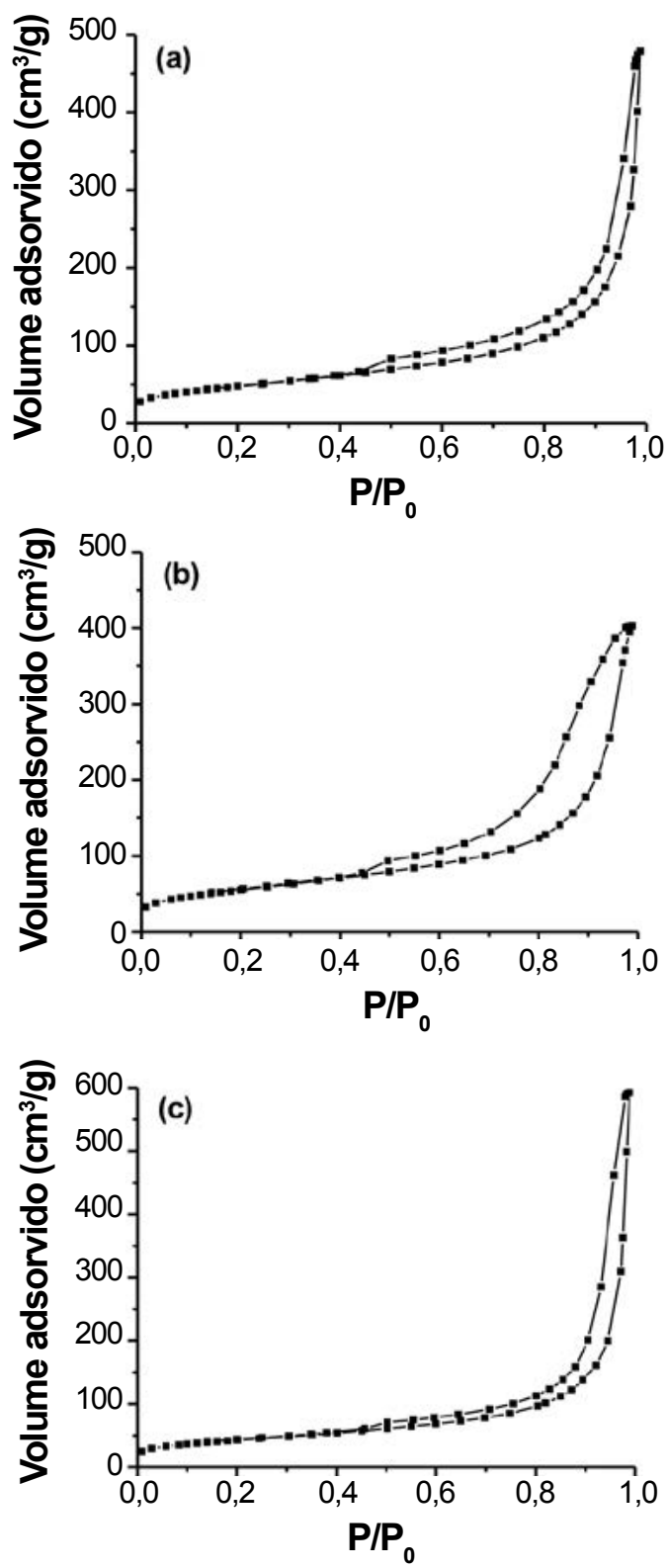

Figura 5: Isoterma de adsorção/dessorção de $\mathrm{N}_{2}$ das amostras sintetizadas: (a) ES1; (b) ES2; (c) ES3.

[Figure 5: $\mathrm{N}_{2}$ adsorption/desorption isotherm of the samples synthesized: (a) ES1; (b) ES2; (c) ES3.]

as amostras apresentam curvas classificadas como H3, que é caracterizada por apresentar diferentes caminhos de evaporação e condensação entre os processos de adsorção e dessorção sofridos pelos materiais adsorventes [19, 20]. As curvas destas amostras (Figs. 5a-c) apresentam um patamar para valores de $P / P_{0}$ entre 0 e 0,4 e depois um ramo de histerese na região de elevada pressão relativa com declividade bastante acentuada, demonstrando assim formação de multicamadas e a presença de mesoporos interparticula, ou seja, poros que se originaram em virtude da aglomeração de pequenos cristalitos [20] apoiando desta forma as análise de MEV. Observa-se ainda para todas as curvas da Fig. 5 que o fenômeno de histerese é mais 
Tabela II - Valores de área específica total BET, área específica equivalente de microporos, área superficial externa e volume de microporos e diâmetro médio de poros das amostras.

[Table II - Values of specific BET surface area, micropores area, external surface area, micropores volume and pore diameter of the samples.]

\begin{tabular}{cccccc}
\hline & $\begin{array}{c}\text { Área } \\
\text { Amostra } \\
\text { BET } \\
\left(\mathrm{m}^{2} / \mathrm{g}\right)\end{array}$ & $\begin{array}{c}\text { Área } \\
\text { microporos } \\
\left(\mathrm{m}^{2} / \mathrm{g}\right)\end{array}$ & $\begin{array}{c}\text { Área } \\
\text { superficial } \\
\text { externa } \\
\left(\mathrm{m}^{2} / \mathrm{g}\right)\end{array}$ & $\begin{array}{c}\text { Diâmetro } \\
\text { médio } \\
\text { de poros } \\
(\mathrm{nm})\end{array}$ & $\begin{array}{c}\text { Volume } \\
\text { microporos } \\
\left(\mathrm{cm}^{3} / \mathrm{g}\right)\end{array}$ \\
\hline ES1 & 171,6 & 10,69 & 160,91 & 10,06 & 0,002968 \\
ES2 & 198,7 & 16,24 & 182,49 & 11,02 & 0,005397 \\
ES3 & 203,4 & 15,30 & 188,13 & 12,49 & 0,005462 \\
\hline
\end{tabular}

acentuado para a amostra ES2, mostrando neste caso uma maior diferença nos processos de evaporação e condensação sugerindo que este material apresenta poros mais rasos e largos em relação às amostras ES1 e ES3 que apresentam poros mais estreitos e profundos.

A Tabela II mostra os resultados extraídos dos ensaios de BET relacionados como a área específica, volume de microporos e tamanho médio de poros dos materiais produzidos. Observa-se que o valor de área específica BET (soma da área superficial externa e área de microporos) das amostras aumenta significativamente de 171,6-198,7 $\mathrm{m}^{2} / \mathrm{g}$ durante períodos de preparação a partir de $44 \mathrm{~h}$ até $90 \mathrm{~h}$, no entanto sobe mais lentamente para os períodos subsequentes $(198,7-$ $203,4 \mathrm{~m}^{2} / \mathrm{g}$ ). Estes valores encontram-se superiores aos obtidos para Zn-estevensita $\left(50-80 \mathrm{~m}^{2} / \mathrm{g}\right)$ sintetizada via hidrólise da uréia [7] sem a utilização de processo hidrotermal. O pequeno aumento da área de superfície para o tempo de $138 \mathrm{~h}$ pode estar relacionada à composição química da folha octaédrica da $\mathrm{Zn}$-estevensita. $\mathrm{O}$ volume de microporos, para as amostras ES2 e ES3, são praticamente iguais apesar das mesmas exibirem certo aumento no diâmetro médio de poros. Isto pode sugerir, conforme comentado anteriormente, que a amostra sintetizada em $90 \mathrm{~h}$ apresenta poros mais largos e rasos e a obtida em $138 \mathrm{~h}$ exibe poros mais estreitos e fundos, resultando desta forma em uma compensação a qual se traduz em áreas específicas semelhantes.

\section{CONCLUSÕES}

Aplicando-se certas modificações ao método da hidrólise da uréia é possível produzir esmectita sintética do tipo $\mathrm{Zn}$ estevensita com espaçamento basal em $\sim 15 \AA$, apresentando o material obtido boa cristalinidade, capacidade de troca de cátions na faixa de 63 a $70 \mathrm{mEq} / 100 \mathrm{~g}$, e área superficial na faixa de 171,6 a $203,4 \mathrm{~m}^{2} / \mathrm{g}$, melhorando-se as propriedades das amostras produzidas com o aumento do tempo de reação. As condições de baixa temperatura de síntese, o gel sem envelhecimento e o processo ocorrendo em sistema estático podem vir a tornar no futuro a metodologia promissora e favorável em termos econômicos se levado à escala industrial pois trata-se de um procedimento simples e de fácil preparação de argila esmectítica.

\section{AGRADECIMENTOS}

Ao Prof. R. S. Angélica (Instituto de Geociências, UFPA) e E. Fagury-Neto (Faculdade de Engenharia de Materiais, UFPA) pela colaboração na realização das análises.

\section{REFERÊNCIAS}

[1] P. Souza Santos, Ciência e Tecnologia de Argilas, 2a Ed., vol.1, Edgar Blucher, S. Paulo, SP (1989) 66.

[2] J. T. Kloprogge, S. Komarneni, J. E. Amonette, Clays Clay Miner. 47 (1999) 529-554.

[3] S. Lantenois, R. Champallier, J. M. Bény, F. Muller, Appl. Clay Sci. 38 (2008) 165-178.

[4] P. Anadão, L. F. Sato, H. Wiebeck, F. R. ValenzuelaDiaz, Appl. Clay Sci. 48 (2010) 127-132.

[5] J. T. Kloprogge, J. Porous Mater. 5 (1998) 5-41.

[6] D. Zhang, C.-H. Zhou, C.-X. Lin, D.-S. Tong, W.-H. Yu, Appl. Clay Sci. 50 (2010) 1-11.

[7] J. C. A. A. Roelofs, P. H. Berben, Appl. Clay Sci. 33 (2006) 13-20.

[8] R. Prihod 'ko, E. J. M. Hensen, M. Sychev, I. Stolyarova, T. E. Shubina, I. Astrelin, R. A. Van Santen, Micropor. Mesopor. Mater. 69 (2004) 49-63.

[9] R. J. M. J. Vogels, J. T. Kloprogge, J. W. Geus, Am. Mineralogist 90 (2005) 931-944.

[10] R. Prihod ko, M. Sychev, E. J. M. Hensen, J. A. R. Van Venn, R. A. Van Santen, Studies Surface Sci. Catal. 142 (2002) 271-278.

[11] H. D. Chapman, Cation exchange capacity, In: C. A. Black et al (Eds.), Methods of soil analysis. Part 2, Am. Soc. Agronomy, Madison, EUA (1965) 891-901.

[12] J. T. Kloprogge, M. Hammond, L. Hickey, R. L. Frost, J. Mater. Sci. Lett. 21 (2002) 931-933.

[13] R. Srivastava, S. Fujita, M. Arai, Appl. Clay Sci. 43 (2009) 1-8.

[14] S. Petit, D. Righi, A. Decarreau, Clays Clay Miner. 56, 6 (2008) 645-654.

[15] N. Takahashi, M. Tanaka, T. Satoh, T. Endo, M. Shimada, Micropor. Mater. 9 (1997) 35-42.

[16] A. A. Khassin, T. M. Yurieva, G. N. Kustova, L. M. Plyasova, T. A. Krieger, I. S. Itenberg, M. P. Demeshkina, T. V. Larina, V. F. Anufrienko, V. N. Parmon. Mat. Res. Innovat. 4 (2001) 251-261.

[17] S. Shimoda, Clay Miner. 9 (1971) 185-192.

[18] A. Benhammou, A. Yaacoubi, L. Nibou, B. Tanouti, J. Colloid Interf. Sci. 282 (2005) 320-326.

[19] K. S. W. Sing, D. H. Everett, R. A. W. Haul, L. Moscou, R. A. Pierotti, J. Rouquerol, T. Siemieniewska, Pure Appl. Chem. 57 (1985) 603-619.

[20] S. Korichi, A. Elias, A. Mefti, A. Bensmaili, Appl. Clay Sci. 59-60 (2012) 76-83.

(Rec.22/11/2012, Rev. 03/11/2013, Ac. 25/01/2014) 